IJEMD-M, 1 (1) (2022) $102-110 \quad$ https://doi.org/10.54938/ijemdm.2022.01.1.2
International Journal of Emerging Multidiciplinaries
Mathematics
Research Paper
Journal Homepage: $\underline{w w w . i j e m d . c o m}$
ISSN (print): $2790-1998$

\title{
Approximate Solution of Generalized Modified $b$-Equation by Optimal Auxiliary Function Method
}

\author{
Rashid Nawaz ${ }^{1}$, Laiq Zada $^{1}$ and Aatif Ali ${ }^{1 *}$ \\ ${ }^{1}$ Department of Mathematics, Abdul Wali Khan University Mardan, 23200, KP, Pakistan \\ *Corresponding author
}

\begin{abstract}
In this study, the implantation of a new semi-analytical method called the optimal auxiliary function method (OAFM) has been extended to partial differential equations. The adopted method was tested upon for approximate solution of generalized modified b-equation. The first-order numerical solution obtained by OAFM has been compared with the variational homotopy perturbation method (VHPM). The method possesses the auxiliary function and control parameters which can be easily handled during simulation of the nonlinear problem. From the numerical and graphical results, we concluded the method is very effective and easy to implement for the nonlinear PDEs.
\end{abstract}

Keywords: Approximate solution, Modified b-Equation, Optimal Auxiliary Function Method (OAFM).

2010 Mathematics Subject Classification: 35C10; 35A24; 35R11

\section{Introduction}

Differential equations (DE) play a vital role in applied science and engineering. PDEs have a variety of applications in optics, hydrodynamics electromagnetism, economics, financial mathematics, and computer science. Usually, nonlinear PDEs don't have the exact solutions, therefore the researchers adopted different approaches for the approximate solution. In such difficult cases, it's so much tough to obtain the exact solution of these nonlinear differential equations. Therefore, a different method has been used in literature for these types of equations [1-5].

In the same research field, we extend the implementation of the OAFM partial differential equations and applied for the approximate solution of modified b-equation. This planed method was introduced by Marinca et.al and used for the solution for the fluid model [6]. Later on, the proposed method was used by researchers for different problems in the field of applied mathematics [7-10]. We aim to apply the proposed method to the generalized modified $b$-equation. The proposed model was introduced by Wazwaz [11] to study a family of 
the important physical equation, namely called modified $b$-equation. The modified b-equation is given as follow,

$$
\frac{\partial \zeta(\eta, t)}{\partial \tau}-\frac{\partial^{3} \zeta(\eta, t)}{\partial \eta^{3} \tau}+(b+1) \zeta^{2}(\eta, t) \frac{\partial \zeta(\eta, t)}{\partial \eta}-b \frac{\partial \zeta(\eta, t)}{\partial \eta} \frac{\partial^{2} \zeta(\eta, t)}{\partial \eta^{2}}-\frac{\partial \zeta(\eta, t)}{\partial \eta} \frac{\partial^{2} \zeta(\eta, t)}{\partial \eta^{2}}=0
$$

In eq. (1) $\mathrm{b}$ is a positive integer. For $\mathrm{b}=3$, eq. (1) is reduced to modified camassa holom (mCH) equation, while, for $b=4$ eq. (1) reduced to modified Degpress ( $\mathrm{mDP}$ ) equation. The modified b-equation has been studied by different methods in the series of papers [12-14].

\section{OAFM Methodology for PDEs}

The extended for of OAFM for partial differential equation has been discussed in the following steps. Let

$$
L[\zeta(\eta, \tau)]=f(\eta, \tau)+N[\zeta(\eta, \tau)]=0,
$$

subject to the boundary condition

$$
\mathrm{B}\left(\zeta, \frac{\partial \zeta}{\partial \tau}\right)
$$

Hence $L, f$ and $\mathrm{N}$ presents a linear operator, a known function, and a nonlinear operator respectively.

Step1: To find the approximate solution of Eq. (2), Let the approximate solution can be expressed in the form of two components given,

$$
\tilde{\zeta}(\eta, \tau)=\zeta_{0}(\eta, \tau)+\zeta_{1}\left(\eta, \tau, C_{i}\right), \quad i=1,2, \ldots \ldots \mathrm{P}
$$

Step 2: To find the initial and the first approximation, we substitute Eq. (4) into Eq. (2), it results in

$$
L\left[\zeta_{0}(\eta, \tau)\right]+L\left[\zeta_{1}\left(\eta, \tau, C_{i}\right)\right]+f(\eta, \tau)+N\left[\zeta_{0}(\eta, \tau)+\zeta_{1}\left(\eta, \tau, C_{i}\right)\right]=0
$$

Step 3: To obtain $\zeta_{0}(\eta, \tau)$ and first order solution $\zeta_{1}(\eta, \tau)$, we consider the following linear equations:

$$
\begin{gathered}
L\left[\zeta_{0}(\eta, \tau)+f(\eta, \tau)\right]=0, \mathrm{~B}\left(\zeta_{0}, \frac{\partial \zeta_{0}}{\partial \tau}\right)=0, \\
L\left[\zeta_{1}\left(\eta, \tau, C_{i}\right)\right]+N\left[\zeta_{0}(\eta, \tau)+\zeta_{1}\left(\eta, \tau, C_{i}\right)\right]=0, \quad \mathrm{~B}\left(\zeta_{1}, \frac{\partial \zeta_{1}}{\partial \tau}\right)=0 .
\end{gathered}
$$

Step 4: The nonlinear term from eq. (7) is expanded in the form of

$$
N\left[\zeta_{0}(\eta, \tau)+\zeta_{1}\left(\eta, \tau, C_{i}\right)\right]=N\left[\zeta_{0}(\eta, \tau)\right]+\sum_{k=1}^{\infty} \frac{\zeta_{1}^{k}}{k !} N^{(k)}\left[\zeta_{0}(\eta, \tau)\right] .
$$

Step 5: Equation (7) is very difficult to solve, so we propose another expression for controlling and accelerating the convergence of the method. So, eq. (7) can be written as

$$
\begin{gathered}
L\left[\zeta_{1}\left(\eta, \tau, C_{i}\right)\right]+\mathrm{A}_{1}\left[\zeta_{0}(\eta, \tau)\right] N\left[\zeta_{0}(\eta, \tau)\right]+\mathrm{A}_{2}\left[\zeta_{0}(\eta, \tau), C_{j}\right]=0, \\
\mathrm{~B}\left(\zeta_{1}, \frac{\partial \zeta_{1}}{\partial \tau}\right)=0,
\end{gathered}
$$

Remark 1: In Eq.(9), $A_{1}$ and $A_{2}$ are known auxiliary functions, that can be chosen based on initial guesses and unknown parameters $C_{i}$ and $C_{j}, i=1,2,3 \ldots, j=s+1, s+2,3, . . \mathrm{p}$. 
Remark 2: $\mathrm{A}_{1}$ and $\mathrm{A}_{2}$ are not unique and are of the same form like $\zeta_{0}(\eta, \tau)$ are the form of $N\left[\zeta_{0}(\eta, \tau)\right]$ or the combination of both $\zeta_{0}(\eta, \tau)$ and $N\left[\zeta_{0}(\eta, \tau)\right]$.

\section{Remark 3:}

(1). If $\zeta_{0}(\eta, \tau)$ or $N\left[\zeta_{0}(\eta, \tau)\right]$ a polynomial function, exponential function, or trigonometric function then the corresponding auxiliary functions should be the sum of polynomial, exponential or trigonometric functions.

(2). If in special case $N\left[\zeta_{0}(\eta, \tau)\right]=0$ then it is clear that $\zeta_{0}(\eta, \tau)$ is an exact solution of Eq. (2).

Step 6: For calculating the $C_{i}$ and $C_{j}$, we use the method of least square, by minimizing the square residual error:

$$
J\left(C_{i}, C_{j}\right)=\int_{0}^{t} \int_{\Omega} R^{2}\left(x, t ; C_{i}, C_{j}\right) d x d t
$$

Hence, $R$ is the residual i.e

$$
R\left(\eta, \tau, C_{i}, C_{j}\right)=L\left[\tilde{\zeta}\left(\eta, \tau, C_{i}, C_{j}\right)\right]+f(\eta, \tau)+N\left[\tilde{\zeta}\left(\eta, \tau, C_{i}, C_{j}\right)\right], i=1,2, . . s, j=S+1, S+2, . . p,
$$

\section{Applications of the Method}

In this section, the adopted method is used for the numerical solution of the $\mathrm{mCH}$ and $\mathrm{DP}$ equation. Additionally, we used the Mathematica 11.0 and Math type for huge computational work.

\subsection{Modified Camassa-Holm Equation (mCH)}

$1^{\text {st }}$ consider Modified Camassa-Holm Equation with initial condition and Exact solution as:

$$
\frac{\partial \zeta(\eta, \tau)}{\partial \tau}-\frac{\partial^{3} \zeta(\eta, \tau)}{\partial \eta^{2} \partial \tau}+3 \zeta^{2} \frac{\partial \zeta(\eta, \tau)}{\partial \eta}-2 \frac{\partial \zeta(\eta, \tau)}{\partial \eta} \frac{\partial^{2} \zeta(\eta, \tau)}{\partial \eta^{2}}-\zeta \frac{\partial^{2} \zeta(\eta, \tau)}{\partial \eta^{2}}=0
$$

subject to initial condition

$$
\zeta(\eta, 0)=-2 \operatorname{sech}^{2}\left(\frac{1}{2} \eta\right)
$$

In eq. (12) linear and nonlinear are given as

$$
\begin{aligned}
& L[\zeta(\eta, \tau)]=\frac{\partial \zeta(\eta, \tau)}{\partial \tau} . \\
& f[(\eta, \tau)]=0 . \\
& N[\zeta]=-\frac{\partial^{3} \zeta}{\partial x^{2} \partial t}+3 \zeta^{2} \frac{\partial \zeta}{\partial x}-2 \frac{\partial \zeta}{\partial x} \frac{\partial^{2} \zeta}{\partial x^{2}}-\zeta \frac{\partial^{2} \zeta}{\partial x^{2}} .
\end{aligned}
$$

The initial approximate $\zeta_{0}(\eta, \tau)$ is obtained from eq. (6).

$$
\frac{\partial \zeta_{0}(\eta, \tau)}{\partial \tau}=0, \zeta_{0}(\eta, 0)=-2 \operatorname{sech}^{2}\left(\frac{1}{2} \eta\right)
$$


The solution of eq. (4.4) is

$$
\zeta_{0}(\eta, \tau)=-2 \operatorname{sech}^{2}\left(\frac{1}{2} \eta\right)
$$

use eq. (18) into eq. (16), the nonlinear operator becomes

$$
\begin{aligned}
& N\left[\zeta_{0}(\eta, \tau)\right]=24 \operatorname{sech}^{6}\left(\frac{\eta}{2}\right) \tanh \left(\frac{\eta}{2}\right)-4 \operatorname{sech}^{2}\left(\frac{\eta}{2}\right) \tanh \left(\frac{\eta}{2}\right)\left(\operatorname{sech}^{4}\left(\frac{\eta}{2}\right)-2 \operatorname{sech}^{2}\left(\frac{\eta}{2}\right) \tanh ^{2}\left(\frac{\eta}{2}\right)\right) \\
& +2 \operatorname{sech}^{2}\left(\frac{\eta}{2}\right)\left(-4 \operatorname{sech}^{4}\left(\frac{\eta}{2}\right) \tanh \left(\frac{\eta}{2}\right)+2 \operatorname{sech}^{2}\left(\frac{\eta}{2}\right) \tanh ^{3}\left(\frac{\eta}{2}\right)\right) .
\end{aligned}
$$

The first approximation $\zeta_{1}(\eta, \tau)$ is given by eq. (9)

$$
\begin{gathered}
\frac{\partial \zeta_{1}(\eta, \tau)}{\partial \tau}+\mathrm{A}_{1}\left[\zeta_{0}(\eta, \tau)\right] N\left[\zeta_{0}(\eta, \tau)\right]+\mathrm{A}_{2}\left[\zeta_{0}(\eta, \tau), C_{j}\right]=0 \\
\zeta_{1}(\eta, 0)=0
\end{gathered}
$$

Here we choose $A_{1}$ and $A_{2}$ as

$$
\left\{\begin{array}{l}
\mathrm{A}_{1}=C_{1}\left[\operatorname{sech}\left(\frac{\eta}{2}\right)\right]^{2}+C_{2}\left[\operatorname{sech}\left(\frac{\eta}{2}\right)\right]^{4} \\
\mathrm{~A}_{2}=C_{3}\left[\operatorname{sech}\left(\frac{\eta}{2}\right)\right]^{6}+C_{4}\left[\operatorname{sech}\left(\frac{\eta}{2}\right)\right]^{8} .
\end{array}\right.
$$

Using eq. (19), and (21) into Eq. (18), we get the first approximation as

$$
\begin{aligned}
& \zeta_{1}(\eta, \tau)=-\tau C_{3} \operatorname{sech}^{6}\left(\frac{\eta}{2}\right)+\tau C_{4} \operatorname{sech}^{8}\left(\frac{\eta}{2}\right)+12 \tau C_{1} \operatorname{sech}^{6}\left(\frac{\eta}{2}\right) \operatorname{sech}^{2}\left(\frac{\eta}{2}\right) \tanh \left(\frac{\eta}{2}\right) \\
& +12 \tau C_{2} \operatorname{sech}^{6}\left(\frac{\eta}{2}\right) \operatorname{sech}^{4}\left(\frac{\eta}{2}\right) \tanh \left(\frac{\eta}{2}\right)+12 \tau C_{1} \operatorname{sech}^{4}\left(\frac{\eta}{2}\right) \operatorname{sech}^{2}\left(\frac{\eta}{2}\right) \tanh ^{3}\left(\frac{\eta}{2}\right) .
\end{aligned}
$$

Adding eq. (18) and eq. (22) we get $1^{\text {st }}$ order approximate solution as

$$
\begin{aligned}
& \tilde{\zeta}(\eta, \tau)=-2 \operatorname{sech}^{2}\left(\frac{1}{2} \eta\right)+-\tau C_{3} \operatorname{sech}^{6}\left(\frac{\eta}{2}\right)+\tau C_{4} \operatorname{sech}^{8}\left(\frac{\eta}{2}\right)+12 \tau C_{1} \operatorname{sech}^{6}\left(\frac{\eta}{2}\right) \operatorname{sech}^{2}\left(\frac{\eta}{2}\right) \\
& \tanh \left(\frac{\eta}{2}\right)+12 \tau C_{2} \operatorname{sech}^{6}\left(\frac{\eta}{2}\right) \operatorname{sech}^{4}\left(\frac{\eta}{2}\right) \tanh \left(\frac{\eta}{2}\right)+12 \tau C_{1} \operatorname{sech}^{4}\left(\frac{\eta}{2}\right) \operatorname{sech}^{2}\left(\frac{\eta}{2}\right) \tanh ^{3}\left(\frac{\eta}{2}\right) .
\end{aligned}
$$

\subsection{Modified Degasperis-Procesi (mDP)}

First consider Modified Degasperis-Procesi with initial condition and exact solution as:

$$
\frac{\partial \zeta(\eta, \tau)}{\partial \tau}-\frac{\partial^{3} \zeta(\eta, \tau)}{\partial \eta^{2} \partial \tau}+4 \zeta^{2} \frac{\partial \zeta(\eta, \tau)}{\partial \eta}-3 \frac{\partial \zeta(\eta, \tau)}{\partial \eta} \frac{\partial^{2} \zeta(\eta, \tau)}{\partial \eta^{2}}-\zeta \frac{\partial^{2} \zeta(\eta, \tau)}{\partial \eta^{2}}=0
$$

subject to initial condition,

$$
\zeta(\eta, 0)=-\frac{15}{8} \operatorname{sech}^{2}\left(\frac{1}{2} \eta\right)
$$

Terms to be consider in eq. (24) as, 


$$
\begin{aligned}
& L[\zeta(\eta, \tau)]=\frac{\partial \zeta(\eta, \tau)}{\partial \tau}, \\
& N[\zeta(\eta, \tau)]=-\frac{\partial^{3} \zeta}{\partial \eta^{2} \partial \tau}+4 \zeta^{2} \frac{\partial \zeta}{\partial \eta}-3 \frac{\partial \zeta}{\partial \eta} \frac{\partial^{2} \zeta}{\partial \eta^{2}}-\zeta \frac{\partial^{2} \zeta}{\partial \eta^{2}}
\end{aligned}
$$

The initial approximate $\zeta_{0}(\eta, \tau)$ is obtained from eq. (6)

$$
\frac{\partial \zeta_{0}(\eta, t)}{\partial \tau}=0, \quad \zeta_{0}(\eta, 0)=-\frac{15}{8} \operatorname{sech}^{2}\left(\frac{1}{2} \eta\right) .
$$

The solution of eq. (28) is

$$
\zeta_{0}(\eta, t)=-\frac{15}{8} \operatorname{sech}^{2}\left(\frac{1}{2} \eta\right) .
$$

use eq. (29) into eq. (27), the nonlinear operator becomes,

$$
\begin{gathered}
N\left[\zeta_{0}(\eta, t)\right]=\frac{3375}{128} \operatorname{sech}^{6}\left(\frac{\eta}{2}\right) \tanh \left(\frac{\eta}{2}\right)-\frac{45}{8} \operatorname{sech}^{2}\left(\frac{\eta}{2}\right) \tanh \left(\frac{\eta}{2}\right) \\
\left(\frac{15}{16} \operatorname{sech}^{4}\left(\frac{\eta}{2}\right)-\frac{15}{8} \operatorname{sech}^{2}\left(\frac{\eta}{2}\right) \tanh ^{2}\left(\frac{\eta}{2}\right)\right)+\frac{15}{8} \operatorname{sech}^{2}\left(\frac{\eta}{2}\right) \\
\left(-\frac{15}{4} \operatorname{sech}^{4}\left(\frac{\eta}{2}\right) \tanh \left(\frac{\eta}{2}\right)+\frac{15}{8} \operatorname{sech}^{2}\left(\frac{\eta}{2}\right) \tanh ^{3}\left(\frac{\eta}{2}\right)\right) .
\end{gathered}
$$

The first approximation $\zeta_{1}(\eta, \tau)$ is given by eq. (9)

$$
\begin{gathered}
\frac{\partial \zeta_{1}(\eta, \tau)}{\partial \tau}+\mathrm{A}_{1}\left[\zeta_{0}(\eta, \tau)\right] N\left[\zeta_{0}(\eta, \tau)\right]+\mathrm{A}_{2}\left[\zeta_{0}(\eta, \tau), C_{j}\right]=0, \\
\zeta_{1}(\eta, 0)=0,
\end{gathered}
$$

Here we again choose $A_{1}$ and $A_{2}$ similar as shown in above problem

$$
\left\{\begin{array}{l}
\mathrm{A}_{1}=C_{1}\left[\operatorname{sech}\left(\frac{\eta}{2}\right)\right]^{2}+C_{2}\left[\operatorname{sech}\left(\frac{\eta}{2}\right)\right]^{4} \\
\mathrm{~A}_{2}=C_{3}\left[\operatorname{sech}\left(\frac{\eta}{2}\right)\right]^{6}+C_{4}\left[\operatorname{sech}\left(\frac{\eta}{2}\right)\right]^{8} .
\end{array}\right.
$$

Using eq. (29), and (30) into Eq. (31), we get the first approximation as

$$
\begin{aligned}
\zeta_{1}(\eta, \tau)= & \tau C_{3} \operatorname{sech}^{6}\left(\frac{\eta}{2}\right)-\tau C_{4} \operatorname{sech}^{8}\left(\frac{\eta}{2}\right)-14.0625 \tau C_{1} \operatorname{sech}^{6}\left(\frac{\eta}{2}\right) \operatorname{sech}^{2}\left(\frac{\eta}{2}\right) \tanh \left(\frac{\eta}{2}\right) \\
& -14.0625 \tau C_{2} \operatorname{sech}^{6}\left(\frac{\eta}{2}\right) \operatorname{sech}^{4}\left(\frac{\eta}{2}\right) \tanh \left(\frac{\eta}{2}\right)-14.0625 \tau C_{1} \operatorname{sech}^{4}\left(\frac{\eta}{2}\right) \operatorname{sech}^{2}\left(\frac{\eta}{2}\right) \\
& \tanh \left(\frac{\eta}{2}\right)-14.0625 \tau C_{2} \operatorname{sech}^{4}\left(\frac{\eta}{2}\right) \operatorname{sech}^{4}\left(\frac{\eta}{2}\right) \tanh \left(\frac{\eta}{2}\right) .
\end{aligned}
$$

Adding eq. (29) and eq. (33) we get $1^{\text {st }}$ order approximate solution as 


$$
\begin{gathered}
\tilde{\zeta}(\eta, \tau)=-\frac{15}{8} \operatorname{sech}^{2}\left(\frac{1}{2} \eta\right)+\tau C_{3} \operatorname{sech}^{6}\left(\frac{\eta}{2}\right)-\tau C_{4} \operatorname{sech}^{8}\left(\frac{\eta}{2}\right)-14.0625 \tau C_{1} \operatorname{sech}^{6}\left(\frac{\eta}{2}\right) \\
\operatorname{sech}^{2}\left(\frac{\eta}{2}\right) \tanh \left(\frac{\eta}{2}\right)-14.0625 \tau C_{2} \operatorname{sech}^{6}\left(\frac{\eta}{2}\right) \operatorname{sech}^{4}\left(\frac{\eta}{2}\right) \tanh \left(\frac{\eta}{2}\right)-14.0625 \tau C_{1} \\
\operatorname{sech}^{4}\left(\frac{\eta}{2}\right) \operatorname{sech}^{2}\left(\frac{\eta}{2}\right) \tanh \left(\frac{\eta}{2}\right)-14.0625 \tau C_{2} \operatorname{sech}^{4}\left(\frac{\eta}{2}\right) \operatorname{sech}^{4}\left(\frac{\eta}{2}\right) \tanh \left(\frac{\eta}{2}\right) .
\end{gathered}
$$

\section{Numerical Results}

We exhibit the correctness of our process for the provided equations and comparison with the VHPM for different values $\tau$.

Result (1). To find the values of unknown parameters $C_{i}, i=1,2,3$.. we used the collection method.

$$
\begin{aligned}
& C_{1}=0.4921659704908877, C_{2}=-0.2606759872371297, \\
& C_{3}=-0.05781845184781508, C_{4}=-0.10563165777129231
\end{aligned}
$$

By substituting these values in Eq. (23), we obtained the first-order solution for the $\mathrm{mCH}$ equation.

Result (2). similarly, we used the collection method for finding values of $C_{i}, i=1,2,3$.. which are given as follow,

$$
\begin{aligned}
& C_{1}=0.3875663632499093, C_{2}=-0.19320905798049545, \\
& C_{3}=-0.46302224355411176, C_{4}=0.014099963170806097 .
\end{aligned}
$$

Using E. (36) eq. (34), we obtained the first-order solution for the DP equation.

Numerical values are tabulated for first-order OAFM and VHPM solution for the $\mathrm{mCH}$ equation in tables (1-2) at $\tau=0.01$ and $\tau=0.001$ respectively. Tables (3-4) present the approximate solution of OAFM and VHPM solution for DP equation when $\tau=0.01$ and $\tau=0.001$.

Figures (1-3) present the 3D surfaces of first-order OAFM, VHPM, and exact solution for $\mathrm{mCH}$ equation respectively. Figures (4-6) show the 3D surfaces first order of OAFM, Exact solution, and VHPM solution for $\mathrm{mDP}$ equation respectively. Figures (7-8) show the comparison of 1st order OAFM, VHPM, and exact solution for $\mathrm{mCH}$ and $\mathrm{mDP}$ equation at $\tau=0.1$ respectively. From the tabulated results and graphical we conclude that the OAFM solution is very closed to the exact solution as compared to VHPM.

\section{Table 1.}

Numerical results were obtained by first-order OAFM and VHPM solution $\tau=0.01$ for the solution of the $\mathrm{mCH}$ equation.

\begin{tabular}{|c|c|c|c|c|}
\hline$\eta$ & OAFM & Exact & Abs Error VHPM [12] & Abs Error OAFM \\
\hline \hline-1.0 & -1.56446 & -1.5583 & 0.0197059 & 0.00616137 \\
\hline \hline-0.5 & -1.87269 & -1.87067 & 0.0166079 & 0.0020242 \\
\hline 0.5 & -1.88476 & -1.88908 & 0.0169162 & 0.00432592 \\
\hline 1.0 & -1.57996 & -1.58737 & 0.0198189 & 0.00741897 \\
\hline
\end{tabular}


Table 2.

Numerical results were obtained by first order OAFM and VHPM solution $\tau=0.001$ for the solution of the $\mathrm{mCH}$ equation.

\begin{tabular}{|c|c|c|c|c|}
\hline$\eta$ & OAFM & Exact & Abs Error VHPM [12] & Abs Error OAFM \\
\hline-1.0 & -1.57232 & -1.57144 & 0.00197555 & 0.00061118 \\
\hline \hline-0.5 & -1.8793 & -1.87911 & 0.00167455 & 0.000188656 \\
\hline \hline 0.5 & -1.8805 & -1.88095 & 0.00167764 & 0.000446577 \\
\hline 1.0 & -1.5736 & -1.57435 & 0.00197668 & 0.000747114 \\
\hline
\end{tabular}

Table 3.

Numerical results obtained by first-order OAFM and VHPM solution at $\tau=0.01$ for the solution of the mDP equation.

\begin{tabular}{|c|c|c|c|c|}
\hline$\eta$ & OAFM & Exact & Abs Error VHPM [12] & Abs Error OAFM \\
\hline \hline-1.0 & -1.46494 & -1.45747 & 0.0230771 & 0.00747007 \\
\hline-0.5 & -1.7529 & -1.75151 & 0.019418 & 0.00138805 \\
\hline 0.5 & -1.76468 & -1.77309 & 0.0198696 & 0.00840817 \\
\hline 1.0 & -1.47984 & -1.49154 & 0.0232427 & 0.011701 \\
\hline
\end{tabular}

Table 4.

Numerical results obtained by first order OAFM and VHPM solution at $\tau=0.001$ for the solution of $\mathrm{mDP}$ equation.

\begin{tabular}{|c|c|c|c|c|}
\hline$\eta$ & OAFM & Exact & Abs Error VHPM [12] & Abs Error OAFM \\
\hline-1. & -1.47362 & -1.47289 & 0.00231493 & 0.000739793 \\
\hline-0.5 & -1.76157 & -1.76145 & 0.00196192 & 0.000118684 \\
\hline 0.5 & -1.76247 & -1.7636 & 0.00196643 & 0.000861343 \\
\hline \hline 1. & -1.47511 & -1.47629 & 0.00231658 & 0.00117779 \\
\hline
\end{tabular}

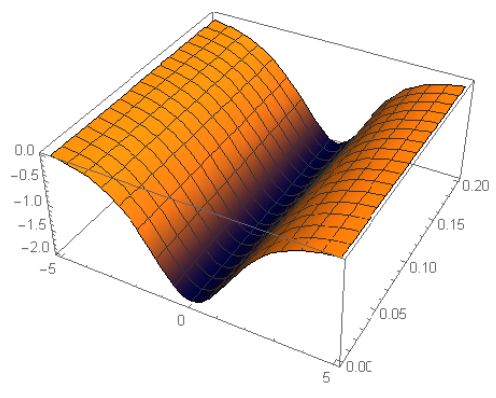

Figure 1: First order OAFM solution for $\mathrm{mCH}$ equation

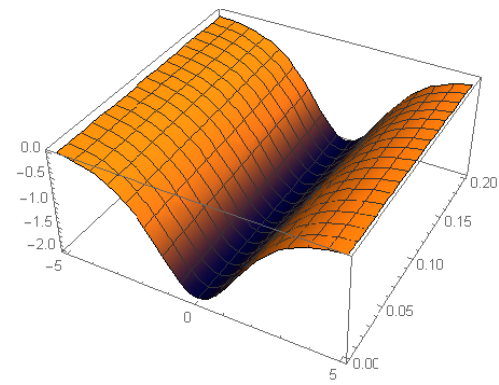

Figure 2: Exact solution for $\mathrm{mCH}$ equation

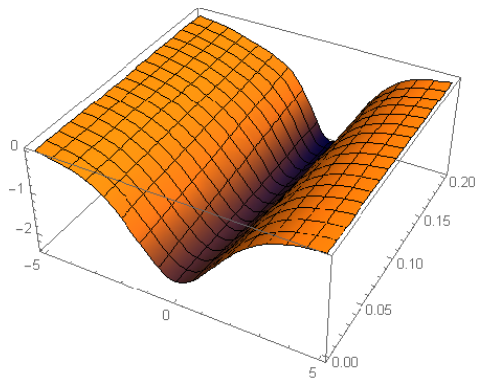

Figure 3: First order VHPM solution for $\mathrm{mCH}$ equation 


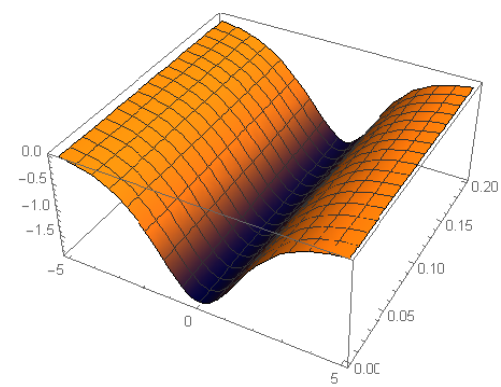

Figure 4: First order OAFM solution for $\mathrm{mDP}$ equation

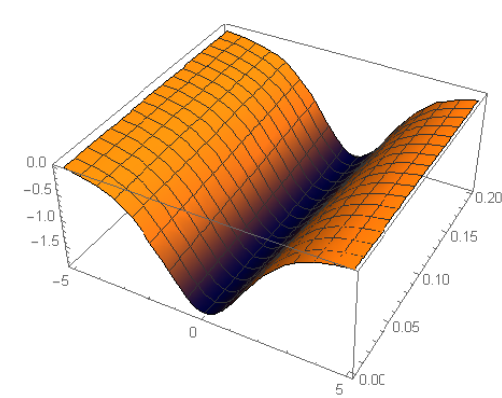

Figure 5: First order OAFM solution for mDP equation

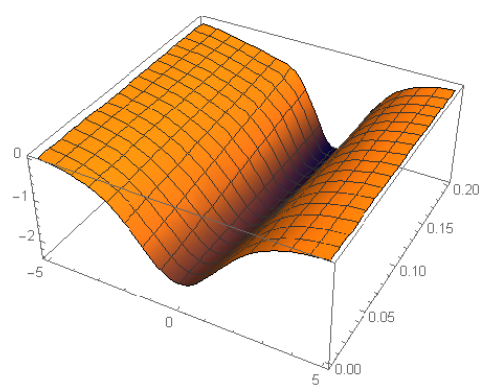

Figure 6: First order OAFM solution for $\mathrm{mDP}$ equation.

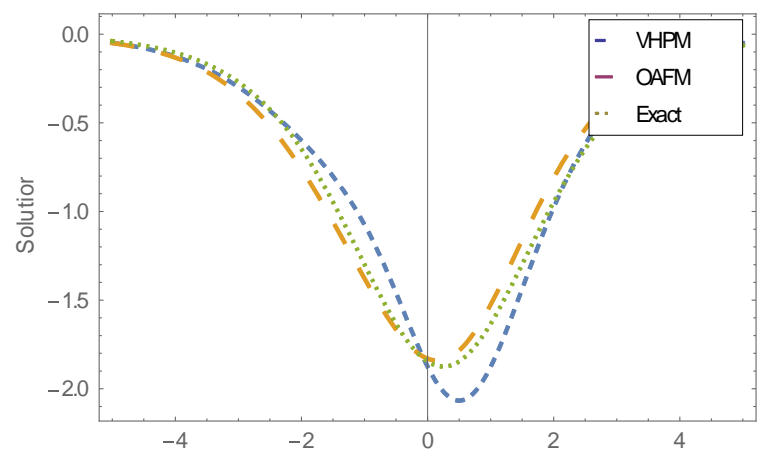

Figure 7: 2D surface are VHPM, OAFM and exact solution at $\tau=$ 0.1 for $\mathrm{mCH}$ equation.

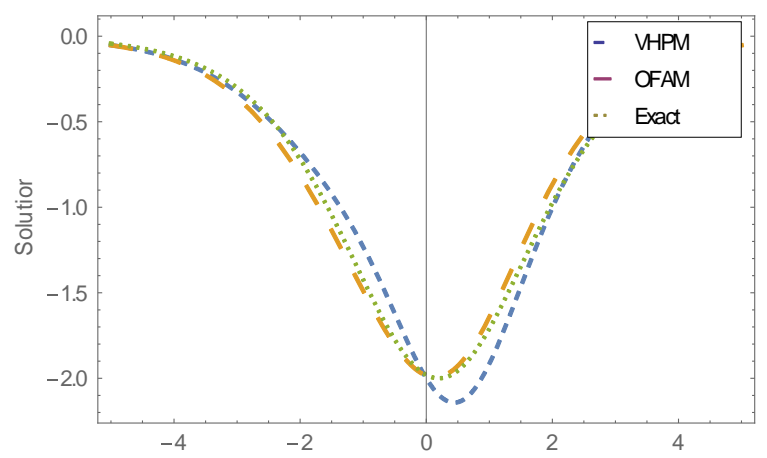

Figure 8: 2D surface are VHPM, OAFM and exact solution at $\tau=$ 0.1 for mDP equation.

\section{Conclusion}

In this work, the suggested method test for the approximate solution of $\mathrm{mCH}$ and $\mathrm{mDP}$ equations. The numerical results have been compared with VHPM. From the results, we conclude that OAFM converges rapidly than VHPM after only one iteration.

\section{Competing Interests}

The author(s) declare no competing interests.

\section{References}

[1] El-Sayed, A. M. A., and M. Gaber. The Adomian decomposition method for solving partial differential equations of fractal order in finite domains. Physics Letters A. 359 (3), 175-182 (2006). 
[2] Dehghan, Mehdi, Jalil Manafian, and Abbas Saadatmandi. Solving nonlinear fractional partial differential equations using the homotopy analysis method. Numerical Methods for Partial Differential Equations: An International Journal. 26 (2), 448-479 (2010).

[3] He, Ji-Huan. Homotopy perturbation method for solving boundary value problems. Physics letters A. 350 (1-2), 87-88 (2006).

[4] Nawaz, Rashid, et al. Optimum solutions of fractional order Zakharov-Kuznetsov equations. Complexity, (2019).

[4] He, Ji-Huan. Variational iteration method-a kind of non-linear analytical technique: some examples. International journal of non-linear mechanics. 34 (4), 699-708 (1999).

[5] Abdou, M. A., and A. A. Soliman. New applications of variational iteration method. Physica D: Nonlinear Phenomena. 211 (1-2), 1-8 (2005).

[6] Marinca, Bogdan, and Vasile Marinca. Approximate analytical solutions for thin film flow of a fourth grade fluid down a vertical cylinder. Proceed Romanian Academy, Series A. 19 (1), 69-76 (2018).

[7] Zada, L., Nawaz, R., Nisar, K. S., Tahir, M., Yavuz, M., Kaabar, M. K., \& Martínez, F. New approximate-analytical solutions to partial differential equations via auxiliary function method. Partial Differential Equations in Applied Mathematics, 4, 100045, (2021).

[8] Zada, Laiq, et al. New algorithm for the approximate solution of generalized seventh order KortewegDevries equation arising in shallow water waves. Results in Physics. 20, 103744 (2021).

[9] Zada, L., et al. A New Approach for Solving Fredholm Integro-Differential Equations. Information Sciences Letters. 10 (3), 3 (2021).

[10] Zada, Laiq, et al. New approximate-analytical solutions to partial differential equations via auxiliary function method. Partial Differential Equations in Applied Mathematics. 4, 100045 (2021).

[11] Wazwaz, Abdul-Majid. Solitary wave solutions for modified forms of Degasperis-Procesi and Camassa-Holm equations. Physics Letters A. 352 (6), 500-504 (2006).

[12] Yousif, Majeed A., Bewar A. Mahmood, and Fadhil H. Easif. A New Analytical Study of Modified Camassa-Holm and Degasperis-Procesi Equations. American Journal of Computational Mathematics, 5 (3), 267-273 (2015).

[13] Zhang, Ben-gong, Shao-yong Li, and Zheng-rong Liu. Homotopy perturbation method for modified Camassa-Holm and Degasperis-Procesi equations. Physics Letters A. 372 (11), 1867-1872 (2008).

[14] Behera, Ratikanta, and Mani Mehra. Approximate solution of modified CamassaHolm and Degasperis-Procesi equations using wavelet optimized finite difference method. International Journal of Wavelets, Multiresolution and Information Processing 11 (02) (2013). 